PROCEEDINGS OF THE

AMERICAN MATHEMATICAL SOCIETY

Volume 139, Number 6, June 2011, Pages 2239-2248

S 0002-9939(2010)10721-9

Article electronically published on November 5, 2010

\title{
ANOSOV THEOREM FOR COINCIDENCES ON SPECIAL SOLVMANIFOLDS OF TYPE (R)
}

\author{
KU YONG HA, JONG BUM LEE, AND PIETER PENNINCKX
}

(Communicated by Alexander N. Dranishnikov)

\begin{abstract}
Suppose that $S$ and $S^{\prime}$ are simply connected solvable Lie groups of type $(\mathrm{R})$ with the same dimension. We show that the Lefschetz coincidence numbers of maps $f, g: \Gamma \backslash S \rightarrow \Gamma^{\prime} \backslash S^{\prime}$ between special solvmanifolds $\Gamma \backslash S \rightarrow$ $\Gamma^{\prime} \backslash S^{\prime}$ can be computed algebraically as follows:

$$
L(f, g)=\operatorname{det}\left(G_{*}-F_{*}\right),
$$

where $F_{*}, G_{*}$ are the matrices, with respect to any preferred bases, of morphisms of Lie algebras induced by $f$ and $g$. This generalizes a recent result by S. W. Kim and J. B. Lee to special solvmanifolds of type (R). Moreover, we can drop the dimension match condition imposed in the latter result.
\end{abstract}

\section{INTRODUCTION}

Let $M$ and $N$ be closed manifolds, and $f, g: M \rightarrow N$ continuous maps. Then we define $\operatorname{Coin}(f, g)=\{x \in M \mid f(x)=g(x)\}$, the coincidence set of $f$ and $g$. Coincidence theory for pairs $f, g$ is a natural extension of fixed point theory for a self-map $f: M \rightarrow M$. There are well-known homotopy invariants in coincidence theory which are the Lefschetz coincidence number $L(f, g)$ and Nielsen coincidence number $N(f, g)$. Suppose that $M, N$ are closed orientable manifolds of the same dimension. Then $L(f, g)$ is defined ([15, Chap. 7]). The Nielsen coincidence number $N(f, g)$ is a non-negative integer with the property that any two maps $f^{\prime}, g^{\prime}$ which are homotopic to $f, g$, respectively, have at least $N(f, g)$ coincidences. The Nielsen coincidence number is much more powerful than the Lefschetz coincidence number but computing it is very hard.

In 4, Brooks, Brown, Pak and Taylor show that for a self-map $f: T^{n} \rightarrow T^{n}$ on a torus $T^{n}$, the Nielsen number $N(f)$ and the Lefschetz number $L(f)$ are equal up to sign, i.e. $N(f)=|L(f)|$. Additionally, they show that $L(f)=\operatorname{det}\left(I-f_{*}\right)$, where $f_{*}: \pi_{1}\left(T^{n}\right) \rightarrow \pi_{1}\left(T^{n}\right)$ is the endomorphism on $\pi_{1}\left(T^{n}\right)$ induced by $f$. In [1], Anosov

Received by the editors August 11, 2009 and, in revised form, May 31, 2010.

2010 Mathematics Subject Classification. Primary 55M20, 54H25, 57S30.

Key words and phrases. Nielsen coincidence number, solvmanifold.

The second-named author is supported partially by the Korea Science and Engineering Foundation (KOSEF) grant funded by the Korea government (MOST) (No. R01-2007-000-10097-0) and by the Sogang University Research Grant of 2010.

The third-named author is supported by a Ph.D. fellowship of the Research FoundationFlanders (FWO).

(C)2010 American Mathematical Society Reverts to public domain 28 years from publication 
generalizes this result to compact nilmanifolds: he proves that $N(f)=|L(f)|$ for any self-map $f$ on a nilmanifold. He also gives a formula for the Lefschetz number $L(f)$. Let $L$ be a connected, simply connected, nilpotent Lie group and $\Gamma$ a uniform lattice of $L$. Then by definition, $M=\Gamma \backslash L$ is a nilmanifold. One can show that any self-map $f: M \rightarrow M$ is homotopic to a map obtained from an endomorphism $F: L \rightarrow L$ for which $F(\Gamma) \subset \Gamma$. Let $F_{*}$ be the homomorphism of Lie algebras induced by $F$. Then Anosov shows that $L(f)=\operatorname{det}\left(I-F_{*}\right)$.

The relation between the Nielsen number and the Lefschetz number for self-maps on nilmanifolds has been generalized to coincidence theory by different authors (14, 16]; see also [5, 9, 12]), who show that the Nielsen coincidence number $N(f, g)$ equals the absolute value of the Lefschetz coincidence number $L(f, g)$ for any pair of maps $f, g: M \rightarrow N$ between nilmanifolds of equal dimension. McCord generalizes this result to special solvmanifolds ([14, Theorem 2.1]). In this way, he reduces the computation of the Nielsen coincidence number of a pair of maps between special solvmanifolds to the computation of the Lefschetz coincidence number. For a long time, however, a practical formula for the Lefschetz coincidence number was only known for maps on the torus ([3]). Only recently, this formula has been generalized to nilmanifolds (12]), but only under the 'dimension match' condition. Let $L, L^{\prime}$ be simply connected, nilpotent Lie groups of equal dimension and $\Gamma \subset L, \Gamma^{\prime} \subset L^{\prime}$ uniform lattices. For any $f, g: \Gamma \backslash L \rightarrow \Gamma^{\prime} \backslash L^{\prime}$, there exist morphisms of Lie groups $\Phi, \Psi: L \rightarrow L^{\prime}$ such that $\Phi$ is a lift of a map homotopic to $f$ and $\Psi$ is a lift of a map homotopic to $g$. Now $\Phi, \Psi: L \rightarrow L^{\prime}$ induce morphisms of Lie algebras $\Phi_{*}, \Psi_{*}: \mathfrak{L} \rightarrow \mathfrak{L}^{\prime}$. The uniform lattices $\Gamma$ and $\Gamma^{\prime}$ give rise to preferred bases for $\mathfrak{L}$ and $\mathfrak{L}^{\prime}$ (a precise definition is in the next section). Let $F_{*}$ and $G_{*}$ be the matrices of the morphisms $\Phi_{*}, \Psi_{*}$ with respect to any preferred bases of $\mathfrak{L}$ and $\mathfrak{L}^{\prime}$. Then it is shown in [12] that

$$
L(f, g)=\operatorname{det}\left(G_{*}-F_{*}\right)
$$

when the 'dimension match' condition is satisfied, that is, when the Lie groups $\gamma_{i}(L)$ and $\gamma_{i}\left(L^{\prime}\right)$ in the lower central series of $L$ and $L^{\prime}$ have equal dimension.

In this paper, we prove that formula (1.1) holds in general, also when the 'dimension match' condition is not satisfied. Additionally, we generalize the formula to special solvmanifolds of type (R). The idea for our proof comes from the proofs of [10, Proposition 2.7] and [15, Corollary 7.25], and [2, p. 398].

\section{SolvmANIFOLDS}

We recall from [6, 10, 11] some definitions about solvable Lie groups and give some basic properties which are necessary for our discussion. A connected solvable Lie group $S$ is said to be of type (NR) (for 'no roots') [11] if the eigenvalues of $\operatorname{Ad}(x): \mathfrak{S} \rightarrow \mathfrak{S}$ are always either equal to 1 or else they are not roots of unity. Solvable Lie groups of type (NR) were considered first in [11. A connected solvable Lie group $S$ is said to be of type (R) (or completely solvable) if $\operatorname{ad}(X): \mathfrak{S} \rightarrow \mathfrak{S}$ has only real eigenvalues for each $X \in \mathfrak{S}$. A connected solvable Lie group $S$ is said to be of type (E) (or exponential) if exp : $\mathfrak{S} \rightarrow S$ is surjective. Below we list some important and relevant properties of connected solvable Lie groups. See [6, 7, 10] for more details. 
(1) Abelian $\Longrightarrow$ Nilpotent $\Longrightarrow$ type (R) $\Longrightarrow$ type (E) $\Longrightarrow$ type (NR).

(2) (Rigidity of Lattices) Let $S$ and $S^{\prime}$ be connected and simply connected solvable Lie groups of type (R), and let $\Gamma$ be a lattice of $S$. Then any homomorphism from $\Gamma$ to $S^{\prime}$ extends uniquely to a Lie group homomorphism from $S$ to $S^{\prime}$.

Let $S$ be a connected and simply connected solvable Lie group. A discrete subgroup $\Gamma$ of $S$ is a lattice of $S$ if $\Gamma \backslash S$ is compact, and in this case, we say that $\Gamma \backslash S$ is a special solvmanifold. For a special solvmanifold, the universal covering space is an orientable Lie group and all covering transformations are orientationpreserving as left translations of this Lie group. Hence any special solvmanifold is orientable. A special solvmanifold $M=\Gamma \backslash S$ is of type (R) if $S$ is of type (R).

Let us first recall the construction of the Chevalley-Eilenberg complex of a Lie algebra $\mathfrak{G}$ : the complex is given by

$$
0 \longrightarrow \bigwedge^{n} \mathfrak{G} \longrightarrow \cdots \longrightarrow \bigwedge^{2} \mathfrak{G} \longrightarrow \mathfrak{G} \longrightarrow \mathbb{R} \longrightarrow 0
$$

with the differential

$$
\partial\left(w_{1} \wedge \cdots \wedge w_{k}\right)=\sum_{i<j}(-1)^{i+j+1}\left[w_{i}, w_{j}\right] \wedge w_{1} \wedge \cdots \wedge \widehat{w}_{i} \wedge \cdots \wedge \widehat{w}_{j} \wedge \cdots \wedge w_{k} .
$$

The exterior algebra $\bigwedge \mathfrak{G}^{*}$ over the dual $\mathfrak{G}^{*}$ of $\mathfrak{G}$ is canonically identified with the dual of $\bigwedge \mathfrak{G}$, and $\bigwedge \mathfrak{G}^{*}$ is a cochain complex with the coboundary operator $\delta$, defined as the transpose of $\partial$.

In what follows, we shall only deal with special solvmanifolds of type $(\mathrm{R})$. We start with the following result of Hattori 8 .

Theorem 2.1. Let $\left(\bigwedge \mathfrak{S}^{*}, \delta\right)$ denote the Chevalley-Eilenberg complex associated to the Lie algebra $\mathfrak{S}$ of a simply connected solvable Lie group $S$ of type (R). If $\Gamma \subset S$ is a lattice, then

$$
H^{*}(\Gamma \backslash S) \cong H^{*}\left(\bigwedge \mathfrak{S}^{*}\right)
$$

Recall that the Hirsch rank of $\Gamma$ is equal to the dimension $n$ of the Lie group $S$. Since $S$ is simply connected of type (R), the exponential map exp : $\mathfrak{S} \rightarrow S$ is a diffeomorphism. We denote the inverse by $\log : S \rightarrow \mathfrak{S}$. We can choose a set of $n$ generators $\left\{\gamma_{i}\right\}$ of $\Gamma$ and write $e_{i}=\log \gamma_{i}$. Then $\left(e_{i}\right)$ is a basis of the vector space $\mathfrak{S}$ and $e_{1} \wedge \cdots \wedge e_{n}$ represents a fundamental class of $H_{n}(\wedge \mathfrak{S})$. We refer to $\left(e_{i}\right)$ as a preferred basis of $\mathfrak{S}$ or of the uniform lattice $\Gamma$.

Let $f: \Gamma \backslash S \rightarrow \Gamma^{\prime} \backslash S^{\prime}$ be a continuous map between special solvmanifolds of type (R) with the same dimension $n$. Take any lifting $\tilde{f}: S \rightarrow S^{\prime}$ of $f$. This lifting induces a homomorphism $\varphi: \Gamma \rightarrow \Gamma^{\prime}$ by the following rule:

$$
\tilde{f} \alpha=\varphi(\alpha) \tilde{f} \text { for } \alpha \in \Gamma \text {. }
$$

By the rigidity of lattices, $\varphi$ extends uniquely to a Lie group homomorphism $F$ : $S \rightarrow S^{\prime}$. Then $F$ induces a map $\phi_{F}: \Gamma \backslash S \rightarrow \Gamma^{\prime} \backslash S^{\prime}$ so that $F$ is a lifting of $\phi_{F}$, and the induced homomorphism between the groups $\Gamma, \Gamma^{\prime}$ of covering transformations is exactly the same as $\varphi$. Hence $f$ is homotopic to $\phi_{F}$. Since we are dealing with homotopy invariants, we shall assume that $f=\phi_{F}$; in other words, $f$ is covered by a Lie group homomorphism $F: S \rightarrow S^{\prime}$. 
Denote by $F_{*}: \mathfrak{S} \rightarrow \mathfrak{S}^{\prime}$ the differential of $F$, and by $F^{*}: \mathfrak{S}^{\prime *} \rightarrow \mathfrak{S}^{*}$ the transpose of $F_{*}$. By Theorem 2.1, we have the commutative diagrams

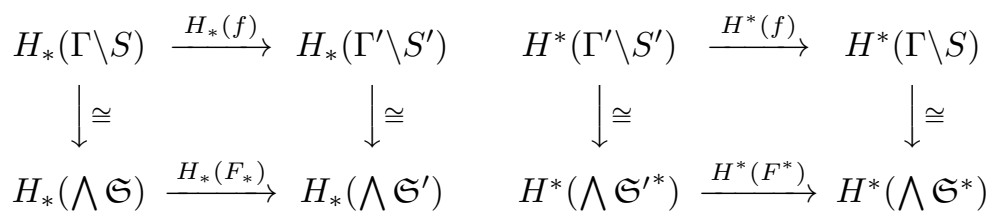

\section{TheOREM}

Let $\Gamma \backslash S$ and $\Gamma^{\prime} \backslash S^{\prime}$ be special solvmanifolds of type (R) with the same dimension $n$. If we use $F_{*}: \mathfrak{S} \rightarrow \mathfrak{S}^{\prime}$ to denote the morphism of Lie algebras associated to $f: \Gamma \backslash S \rightarrow \Gamma^{\prime} \backslash S^{\prime}$ as in the previous section (and similarly for $G_{*}$ ), then we can formulate our main result as follows:

Theorem 3.1. Let $f, g: \Gamma \backslash S \rightarrow \Gamma^{\prime} \backslash S^{\prime}$ be continuous maps. Then

$$
L(f, g)=\operatorname{det}\left(G_{*}-F_{*}\right),
$$

where $F_{*}$ and $G_{*}$ are expressed with respect to any preferred bases of $\mathfrak{S}$ and $\mathfrak{S}^{\prime}$.

For every integer $p>0$, let

$$
F_{p}(n)=\left\{\left(i_{1}, i_{2}, \ldots, i_{p}\right) \mid 1 \leq i_{1}<\cdots<i_{p} \leq n\right\} .
$$

Let $M=\left(a_{i j}\right)$ be an $n \times n$ real matrix. If $I, J \in F_{p}(n)$, then we shall denote by $M_{I, J}$ the square matrix of type $(I, J)$ equal to $\left(a_{i, j}\right)_{(i, j) \in I \times J}$. The determinant $\operatorname{det}\left(M_{I, J}\right)$ is called the minor of the matrix $M$ of indices $I, J$; these determinants are also called the minors of $M$ of order $p$. With this notation:

Lemma 3.2. Let $F: V \rightarrow W$ be a linear map between vector spaces $V$ and $W$ of the same dimension $n$, and let $M$ be a matrix of $F$ with respect to a basis $\left(e_{i}\right)$ of $V$ and a basis $\left(f_{j}\right)$ of $W$. Then, for every integer $p \leq n$, the matrix of $\bigwedge^{p} F: \bigwedge^{p} V \rightarrow \bigwedge^{p} W$ with respect to the basis $\left(e_{I}\right)_{I \in F_{p}(n)}$ of $\bigwedge^{p} V$ and the basis $\left(f_{J}\right)_{J \in F_{p}(n)}$ of $\bigwedge^{p} W$ is the matrix $\left(\operatorname{det}\left(M_{I, J}\right)\right)$ of type $\left(F_{p}(n), F_{p}(n)\right)$.

The matrix $\left(\operatorname{det}\left(M_{I, J}\right)\right)$ of type $\left(F_{p}(n), F_{p}(n)\right)$ is called the $p$-th exterior power of the matrix $M$ and is denoted by $\bigwedge^{p}(M)$. When $p=n, \bigwedge^{n}(M)$ is the matrix with the single element $\operatorname{det} M$. For $I \in F_{p}(n)$, denote by $I^{\prime}$ the complement of $I$ so that $I^{\prime} \in F_{n-p}(n)$. Then:

Lemma 3.3. Let $M, N$ be $n \times n$ matrices. Then

$$
\operatorname{det}(N-M)=\sum_{p=0}^{n}(-1)^{p} \sum_{I, J \in F_{p}(n)} \operatorname{det}\left(M_{I, J}\right) \operatorname{det}\left(N_{I^{\prime}, J^{\prime}}\right) .
$$

Proof of Theorem 3.1. Form the diagram

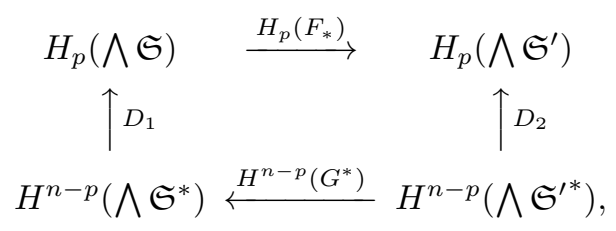

where $D_{1}$ and $D_{2}$ are Poincaré duality isomorphisms. We define

$$
\Theta_{p}=D_{1} \circ H^{n-p}\left(G^{*}\right) \circ D_{2}^{-1} \circ H_{p}\left(F_{*}\right): H_{p}(\bigwedge \mathfrak{S}) \longrightarrow H_{p}\left(\bigwedge \mathfrak{S}^{\prime}\right) .
$$


Then by Theorem 2.1, the Lefschetz coincidence number of $f$ and $g$ is given by

$$
L(f, g)=\sum_{q=0}^{n}(-1)^{q} \operatorname{tr}\left(\Theta_{q}\right) .
$$

Now we choose a preferred basis $\left\{e_{i}\right\}$ for $\mathfrak{S}$ and a preferred basis $\left\{f_{i}\right\}$ for $\mathfrak{S}^{\prime}$ so that $e_{1} \wedge \cdots \wedge e_{n}$ represents a fundamental class of $H_{n}(\wedge \mathfrak{S})$ and $f_{1} \wedge \cdots \wedge f_{n}$ represents a fundamental class of $H_{n}\left(\bigwedge \mathfrak{S}^{\prime}\right)$. Thus the evaluation map $\bigwedge^{p} \mathfrak{S}^{*} \rightarrow$ $\bigwedge^{n-p} \mathfrak{S}$ at $e_{1} \wedge \cdots \wedge e_{n}, e_{I}^{*} \mapsto e_{I^{\prime}}$, is an isomorphism and induces the Poincaré duality isomorphism $D_{1}: H^{p}\left(\bigwedge \mathfrak{S}^{*}\right) \rightarrow H_{n-p}(\bigwedge \mathfrak{S})$. The isomorphism $D_{2}$ can be described similarly.

To evaluate the Lefschetz number, we look at $\Theta_{p}$ on the (co)chain level. Namely, we observe the following commutative diagram of (co)chain complexes that induces $\Theta_{p}:$

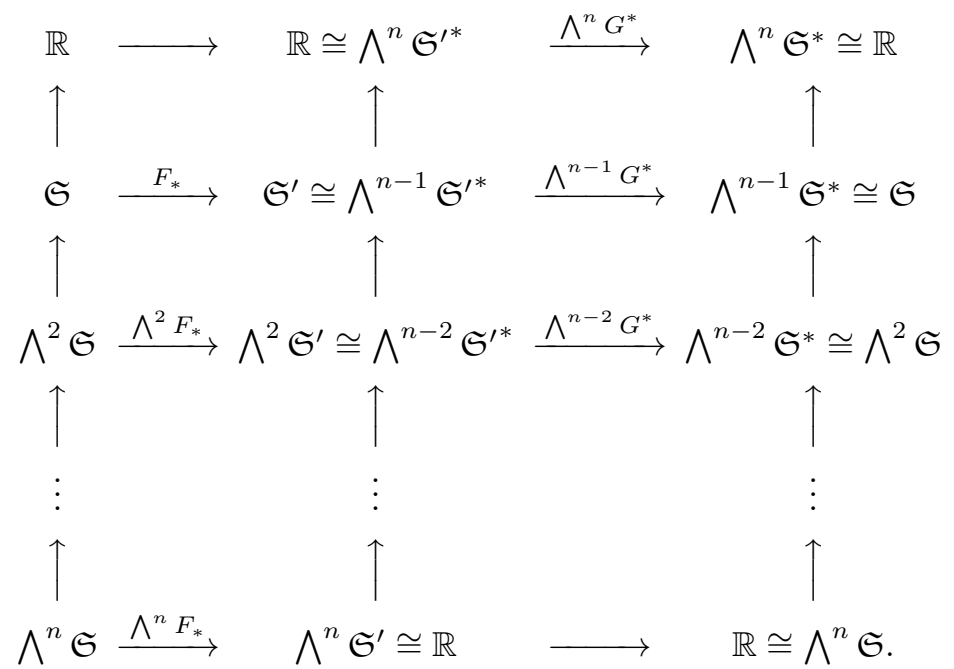

With respect to the fixed bases $\left(e_{i}\right),\left(f_{j}\right)$ of $\mathfrak{S}, \mathfrak{S}^{\prime}$ and the corresponding bases $\left(e_{I}\right)_{I \in F_{p}(n)},\left(f_{J}\right)_{J \in F_{p}(n)}$ of $\bigwedge^{p} \mathfrak{S}, \bigwedge^{p} \mathfrak{S}^{\prime}$, by Lemma 3.2. the matrix of $\bigwedge^{p} F_{*}$ is the $p$-th exterior power of the matrix $F_{*}$, i.e.,

$$
\bigwedge^{p} F_{*}=\left[\operatorname{det}\left(F_{* I, J}\right)\right]_{I, J \in F_{p}(n)} .
$$

Taking the dual, we immediately have that $\bigwedge^{p} F^{*}$ is the transpose of $\bigwedge^{p} F_{*}$. Consequently, the chain map

$$
\bigwedge^{p} \mathfrak{S} \stackrel{\Lambda^{p} F_{*}}{\longrightarrow} \bigwedge^{p} \mathfrak{S}^{\prime} \cong \bigwedge^{n-p} \mathfrak{S}^{*} \stackrel{\Lambda^{n-p} G^{*}}{\longrightarrow} \bigwedge^{n-p} \mathfrak{S}^{*} \cong \bigwedge^{p} \mathfrak{S}
$$

is given by the matrix

$$
\left[\operatorname{det}\left(G_{* I^{\prime}, J^{\prime}}\right)\right]_{I, J \in F_{p}(n)}^{t} \cdot\left[\operatorname{det}\left(F_{* I, J}\right)\right]_{I, J \in F_{p}(n)} .
$$

The trace of this matrix is

$$
\sum_{I, J \in F_{p}(n)} \operatorname{det}\left(F_{* I, J}\right) \operatorname{det}\left(G_{* I^{\prime}, J^{\prime}}\right) .
$$


By the Hopf trace theorem together with Lemma 3.3, we have

$$
\begin{aligned}
L(f, g) & =\sum_{p=0}^{n}(-1)^{p} \operatorname{tr}\left(\Theta_{p}\right)=\sum_{p=0}^{n}(-1)^{p} \operatorname{tr}\left\{\bigwedge^{p} \mathfrak{S} \rightarrow \bigwedge^{p} \mathfrak{S}\right\} \\
& =\sum_{p=0}^{n}(-1)^{p} \sum_{I, J \in F_{p}(n)} \operatorname{det}\left(F_{* I, J}\right) \operatorname{det}\left(G_{* I^{\prime}, J^{\prime}}\right) \\
& =\operatorname{det}\left(G_{*}-F_{*}\right) .
\end{aligned}
$$

Finally, suppose that $\left(e_{i}^{\prime}\right)$ and $\left(f_{j}^{\prime}\right)$ are other bases for $\mathfrak{S}$ and $\mathfrak{S}^{\prime}$, respectively. We note that the transition matrices from one preferred basis to another one (both corresponding to the same fundamental class) have determinant +1 . It follows that $\operatorname{det}\left(G_{*}-F_{*}\right)$ does not depend on the choice between the pairs of preferred bases $\left(e_{i}\right),\left(f_{j}\right)$ and $\left(e_{i}^{\prime}\right),\left(f_{j}^{\prime}\right)$ of $\mathfrak{S}, \mathfrak{S}^{\prime}$. Hence we have completed the proof.

\section{EXAMPLES}

In this section, we illustrate, by some examples, how practical the algebraic coincidence formula is. In the first example, we will consider maps from the threetorus to a three-dimensional nilmanifold. Here the result from [12] cannot be applied since the 'dimension match' condition is not satisfied.

In the second example we will consider self-maps on a three-dimensional solvmanifold. Here, the result from [12] cannot be applied either since [12] only concerns nilmanifolds.

Example 4.1. The Lie group Nil,

$$
\text { Nil }=\left\{\left[\begin{array}{ccc}
1 & x & z \\
0 & 1 & y \\
0 & 0 & 1
\end{array}\right] \mid x, y, z \in \mathbb{R}\right\},
$$

is one of the eight geometries that one considers in the study of 3-manifolds. Its Lie algebra $\mathfrak{n i l}$ is

$$
\mathfrak{n i l}=\left\{\left[\begin{array}{ccc}
0 & a & c \\
0 & 0 & b \\
0 & 0 & 0
\end{array}\right] \mid a, b, c \in \mathbb{R}\right\} .
$$

For any integer $k>0$, we consider the subgroups $\Gamma_{k}$ of Nil:

$$
\Gamma_{k}=\left\{\left[\begin{array}{ccc}
1 & m & \frac{\ell}{k} \\
0 & 1 & n \\
0 & 0 & 1
\end{array}\right] \mid \ell, m, n \in \mathbb{Z}\right\} .
$$

These are uniform lattices of Nil, and every uniform lattice of Nil is isomorphic to some $\Gamma_{k}$.

In what follows, we fix an (ordered) basis

$$
\mathbf{x}=\left[\begin{array}{ccc}
1 & 1 & \frac{0}{k} \\
0 & 1 & 0 \\
0 & 0 & 1
\end{array}\right], \mathbf{y}=\left[\begin{array}{ccc}
1 & 0 & \frac{0}{k} \\
0 & 1 & 1 \\
0 & 0 & 1
\end{array}\right], \mathbf{z}=\left[\begin{array}{ccc}
1 & 0 & \frac{1}{k} \\
0 & 1 & 0 \\
0 & 0 & 1
\end{array}\right]
$$


for $\Gamma_{k}$. Under the logarithmic diffeomorphism log $: \mathrm{Nil} \rightarrow \mathfrak{n i l}$, this basis is mapped to a preferred basis of the vector space nil. This basis is

$$
\log \mathbf{x}=\left[\begin{array}{ccc}
0 & 1 & \frac{0}{k} \\
0 & 0 & 0 \\
0 & 0 & 0
\end{array}\right], \log \mathbf{y}=\left[\begin{array}{ccc}
0 & 0 & \frac{0}{k} \\
0 & 0 & 1 \\
0 & 0 & 0
\end{array}\right], \log \mathbf{z}=\left[\begin{array}{ccc}
0 & 0 & \frac{1}{k} \\
0 & 0 & 0 \\
0 & 0 & 0
\end{array}\right] .
$$

Let $f, g: \mathbb{Z}^{3} \backslash \mathbb{R}^{3} \rightarrow \Gamma_{k} \backslash$ Nil be maps. Using the construction exposed at the end of Section 2, we may assume that $f$ has a lift $F: \mathbb{R}^{3} \rightarrow$ Nil that is a morphism of Lie groups so that $F\left(\mathbb{Z}^{3}\right) \subset \Gamma_{k}$ and that $g$ has a lift $G: \mathbb{R}^{3} \rightarrow$ Nil that is a morphism of Lie groups so that $G\left(\mathbb{Z}^{3}\right) \subset \Gamma_{k}$. Denote by $\varphi: \mathbb{Z}^{3} \rightarrow \Gamma_{k}$ the restriction of $F$. We find all the possible homomorphisms $\varphi: \mathbb{Z}^{3} \rightarrow \Gamma_{k}$. To do so, choose $e_{1}=(1,0,0)$, $e_{2}=(0,1,0)$ and $e_{3}=(0,0,1)$ as a preferred basis for $\mathbb{Z}^{3}$. Let $F_{*}: \mathbb{R}^{3} \rightarrow$ nil be the morphism of Lie algebras induced by $F: \mathbb{R}^{3} \rightarrow$ Nil. For $i=1,2,3$, write

$$
F_{*}\left(e_{i}\right)=a_{i} \log \mathbf{x}+b_{i} \log \mathbf{y}+c_{i} \log \mathbf{z} .
$$

Then

$$
\begin{array}{r}
F\left(e_{i}\right)=\exp \left(F_{*}\left(\log \left(e_{i}\right)\right)\right)=\exp \left(F_{*}\left(e_{i}\right)\right)=\exp \left(a_{i} \log \mathbf{x}+b_{i} \log \mathbf{y}+c_{i} \log \mathbf{z}\right) \\
=\exp \left(\left[\begin{array}{ccc}
0 & a_{i} & \frac{c_{i}}{k} \\
0 & 0 & b_{i} \\
0 & 0 & 0
\end{array}\right]\right)=\left[\begin{array}{ccc}
1 & a_{i} & \frac{c_{i}}{k}+\frac{a_{i} b_{i}}{2} \\
0 & 1 & b_{i} \\
0 & 0 & 1
\end{array}\right] .
\end{array}
$$

Because $F\left(e_{i}\right) \in \Gamma_{k}$, we have that $a_{i}, b_{i} \in \mathbb{Z}$ and $c_{i}+k \frac{a_{i} b_{i}}{2} \in \mathbb{Z}$. Write $z_{i}=c_{i}+k \frac{a_{i} b_{i}}{2}$. Then $z_{i} \in \mathbb{Z}$ and $c_{i}=z_{i}-k \frac{a_{i} b_{i}}{2}$.

Because $F_{*}$ is a morphism of Lie algebras,

$$
\begin{aligned}
& 0=F_{*}\left(\left[e_{1}, e_{2}\right]\right)=\left[F_{*}\left(e_{1}\right), F_{*}\left(e_{2}\right)\right] \\
&=\left[a_{1} \log \mathbf{x}+b_{1} \log \mathbf{y}+c_{1} \log \mathbf{z}, a_{2} \log \mathbf{x}+b_{2} \log \mathbf{y}+c_{2} \log \mathbf{z}\right] \\
& \quad=k\left(a_{1} b_{2}-a_{2} b_{1}\right) \log \mathbf{z} .
\end{aligned}
$$

Hence $\left(a_{1}, b_{1}\right)$ and $\left(a_{2}, b_{2}\right)$ are linearly dependent. One shows that $\left(a_{1}, b_{1}\right),\left(a_{2}, b_{2}\right)$ and $\left(a_{3}, b_{3}\right)$ are linearly dependent. Let $V$ be the subspace of $\mathbb{R}^{2}$ spanned by $\left(a_{1}, b_{1}\right)$, $\left(a_{2}, b_{2}\right)$ and $\left(a_{3}, b_{3}\right)$ and let $(a, b)$ be a generator of the group $V \cap \mathbb{Z}^{2}$ which is cyclic. Then there exist $d_{1}, d_{2}, d_{3} \in \mathbb{Z}$ such that $a_{i}=d_{i} a$ and $b_{i}=d_{i} b$. We see that there exist $a, b, d_{1}, d_{2}, d_{3}, z_{1}, z_{2}, z_{3} \in \mathbb{Z}$ such that for $i=1,2,3$,

$$
F_{*}\left(e_{i}\right)=d_{i} a \log \mathbf{x}+d_{i} b \log \mathbf{y}+\left(z_{i}-k \frac{d_{i}^{2} a b}{2} \log \mathbf{z}\right) .
$$

Hence with respect to the preferred bases $\left\{e_{1}, e_{2}, e_{3}\right\}$ of $\mathbb{R}^{3}$ and $\{\log \mathbf{x}, \log \mathbf{y}, \log \mathbf{z}\}$ of nil, the matrix of $F_{*}$ is given by

$$
F_{*}=\left[\begin{array}{ccc}
d_{1} a & d_{2} a & d_{3} a \\
d_{1} b & d_{2} b & d_{3} b \\
z_{1}-k \frac{d_{1}^{2} a b}{2} & z_{2}-k \frac{d_{2}^{2} a b}{2} & z_{3}-k \frac{d_{3}^{2} a b}{2}
\end{array}\right],
$$

where $a, b, d_{1}, d_{2}, d_{3}, z_{1}, z_{2}, z_{3} \in \mathbb{Z}$. Conversely, one can show that any such matrix corresponds to a morphism of Lie groups $F: \mathbb{R}^{3} \rightarrow$ nil that restricts to a morphism $\varphi: \mathbb{Z}^{3} \rightarrow \Gamma_{k}$.

Therefore, by Theorem 3.1, the Lefschetz coincidence number of $f, g$ is given by

$$
L(f, g)=\operatorname{det}\left(G_{*}-F_{*}\right),
$$


where $F_{*}$ is a matrix as described above and $G_{*}$ is similar.

Example 4.2. The solvable Lie group Sol is also one of the eight geometries. One can describe Sol as a semi-direct product $\mathbb{R}^{2} \rtimes_{\varphi} \mathbb{R}$ where $t \in \mathbb{R}$ acts on $\mathbb{R}^{2}$ via the map

$$
\varphi(t)=\left[\begin{array}{cc}
e^{t} & 0 \\
0 & e^{-t}
\end{array}\right]
$$

Its Lie algebra $\mathfrak{s o l}$ is given as $\mathfrak{s o l}=\mathbb{R}^{2} \rtimes_{\sigma} \mathbb{R}$, where

$$
\sigma(t)=\left[\begin{array}{rr}
t & 0 \\
0 & -t
\end{array}\right]
$$

The Lie group Sol can be embedded into Aff(3) as

$$
\left[\begin{array}{cccc}
e^{t} & 0 & 0 & x \\
0 & e^{-t} & 0 & y \\
0 & 0 & 1 & t \\
0 & 0 & 0 & 1
\end{array}\right]
$$

where $x, y$ and $t$ are real numbers, and hence its Lie algebra $\mathfrak{s o l}$ is isomorphic to the algebra of matrices

$$
\left[\begin{array}{rrrr}
t & 0 & 0 & a \\
0 & -t & 0 & b \\
0 & 0 & 0 & t \\
0 & 0 & 0 & 0
\end{array}\right]
$$

Note that Sol is of type $(\mathrm{R})$. Let

$$
\Gamma=\left\langle a, b, s \mid[a, b]=1, s^{-1}=a^{\ell_{11}} b^{\ell_{21}}, s b s^{-1}=a^{\ell_{12}} b^{\ell_{22}}\right\rangle
$$

be a lattice of Sol. By [13, Corollary 2.2], there are linearly independent vectors $\left(x_{1}, y_{1}\right),\left(x_{2}, y_{2}\right)$ in $\mathbb{R}^{2}$ and a nonzero number $t_{0} \in \mathbb{R}$ so that they satisfy

$$
\left[\begin{array}{ll}
x_{1} & x_{2} \\
y_{1} & y_{2}
\end{array}\right]^{-1}\left[\begin{array}{cc}
e_{0}^{t} & 0 \\
0 & e^{-t_{0}}
\end{array}\right]=\left[\begin{array}{ll}
\ell_{11} & \ell_{12} \\
\ell_{21} & \ell_{22}
\end{array}\right]\left[\begin{array}{ll}
x_{1} & x_{2} \\
y_{1} & y_{2}
\end{array}\right]^{-1}
$$

In this case, $\Gamma$ is embedded in Sol as follows:

$$
a=\left[\begin{array}{cccc}
1 & 0 & 0 & x_{1} \\
0 & 1 & 0 & y_{1} \\
0 & 0 & 1 & 0 \\
0 & 0 & 0 & 1
\end{array}\right], b=\left[\begin{array}{cccc}
1 & 0 & 0 & x_{2} \\
0 & 1 & 0 & y_{2} \\
0 & 0 & 1 & 0 \\
0 & 0 & 0 & 1
\end{array}\right], s=\left[\begin{array}{cccc}
e^{t_{0}} & 0 & 0 & 0 \\
0 & e^{-t_{0}} & 0 & 0 \\
0 & 0 & 1 & t_{0} \\
0 & 0 & 0 & 1
\end{array}\right]
$$

Let $F:$ Sol $\rightarrow$ Sol be a Lie group homomorphism of Sol sending $\Gamma$ into $\Gamma$ itself. Then by [13, Theorem 2.4], $F$ is one of the following:

$$
\begin{aligned}
& F(a)=a^{u} b^{\frac{\ell_{21}}{\ell_{12}}}, F(b)=a^{v} b^{u+\frac{\ell_{22}-\ell_{11}}{\ell_{12}} v}, F(s)=a^{p} b^{q} s \\
& F(a)=a^{-u} b^{v}, F(b)=a^{\frac{\ell_{11}-\ell_{22}}{\ell_{21}} u-\frac{\ell_{12}}{\ell_{21}} v} b^{u}, F(s)=a^{p} b^{q} s^{-1} \\
& F(a)=1, F(b)=1, F(s)=a^{p} b^{q} s^{m} \text { with } m \neq \pm 1
\end{aligned}
$$


for some integer powers. This $F$ induces a map $\phi_{F}: \Gamma \backslash$ Sol $\rightarrow \Gamma \backslash$ Sol so that the following diagram commutes:

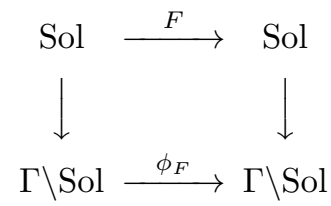

Write $\mathbf{e}_{1}=\log a, \mathbf{e}_{2}=\log b, \mathbf{e}_{3}=\log s$. Then $\left\{\mathbf{e}_{1}, \mathbf{e}_{2}, \mathbf{e}_{3}\right\}$ is a preferred basis of $\mathfrak{s o l}$; they are

$$
\mathbf{e}_{1}=\left[\begin{array}{cccc}
0 & 0 & 0 & x_{1} \\
0 & 0 & 0 & y_{1} \\
0 & 0 & 0 & 0 \\
0 & 0 & 0 & 0
\end{array}\right], \mathbf{e}_{2}=\left[\begin{array}{llll}
0 & 0 & 0 & x_{2} \\
0 & 0 & 0 & y_{2} \\
0 & 0 & 0 & 0 \\
0 & 0 & 0 & 0
\end{array}\right], \mathbf{e}_{3}=\left[\begin{array}{rrrr}
t_{0} & 0 & 0 & 0 \\
0 & -t_{0} & 0 & 0 \\
0 & 0 & 0 & t_{0} \\
0 & 0 & 0 & 0
\end{array}\right] .
$$

With respect to this basis, the induced differential $F_{*}: \mathfrak{s o l} \rightarrow \mathfrak{s o l}$ can be expressed by

$$
\begin{aligned}
& {\left[\begin{array}{ccr}
u & v & * \\
\frac{\ell_{21}}{\ell_{12}} v & u+\frac{\ell_{22}-\ell_{11}}{\ell_{12}} v & * \\
0 & 0 & 1
\end{array}\right] ;} \\
& {\left[\begin{array}{ccr}
-u & \frac{\ell_{11}-\ell_{22}}{\ell_{21}} u-\frac{\ell_{12}}{\ell_{21}} v & * \\
v & u & * \\
0 & 0 & -1
\end{array}\right] ;} \\
& {\left[\begin{array}{ccc}
0 & 0 & * \\
0 & 0 & * \\
0 & 0 & m
\end{array}\right] .}
\end{aligned}
$$

Therefore by Theorem 3.1, the Lefschetz coincidence number and the Nielsen coincidence number of the maps $\phi_{F}, \phi_{G}: \Gamma \backslash$ Sol $\rightarrow \Gamma \backslash$ Sol are

$$
L\left(\phi_{F}, \phi_{G}\right)=\operatorname{det}\left(G_{*}-F_{*}\right), \quad N\left(\phi_{F}, \phi_{G}\right)=\left|\operatorname{det}\left(G_{*}-F_{*}\right)\right|,
$$

where $F_{*}, G_{*}$ are the matrices of types (I), (II), or (III) according to whether $\phi_{F}, \phi_{G}$ are of types (I), (II), or (III).

\section{REFERENCES}

[1] D. V. Anosov, The Nielsen numbers of maps of nil-manifolds, Uspehi Mat. Nauk, 40 (1985), 133-134; Russian Math. Survey, 40 (1985), 149-150. MR807725 (87c:55001)

[2] G. E. Bredon, Topology and Geometry, Springer-Verlag, New York, 1993. MR1224675 (94d:55001)

[3] R. B. S. Brooks, Coincidences, Roots and Fixed Points, PhD thesis, University of California, Los Angeles, 1967

[4] R. B. S. Brooks, R. F. Brown, J. Pak and D. H. Taylor, Nielsen numbers of maps of tori, Proc. Amer. Math. Soc., 52 (1975), 398-400. MR0375287 (51:11483)

[5] R. B. S. Brooks and P. Wong, On changing fixed points and coincidences to roots, Proc. Amer. Math. Soc., 115 (1992), 527-533. MR.1098397 (92k:55002)

[6] K. Dekimpe, K. B. Lee and F. Raymond, Bieberbach theorems for solvable Lie groups, Asian J. Math., 5 (2001), 499-508. MR1868577 (2002h:22011)

[7] V. V. Gorbacevič, Discrete subgroups of solvable Lie groups of type (R), Math. USSR Sbornik, 14 (1971), 238-255. MR0289711 (44:6899)

[8] A. Hattori, Spectral sequence in the de Rham cohomology of fibre bundles, J. Fac. Sci. Univ. Tokyo Sect. I, 8 (1960), 289-331. MR0124918 (23:A2226) 
[9] J. Jezierski, The Nielsen number product formula for coincidences, Fund. Math., 134 (1989), 183-212. MR1071665 (91i:55002)

[10] J. Jezierski, J. Kędra and W. Marzantowicz, Homotopy minimal periods for $N R$-solvmanifolds maps, Topology Appl., 144 (2004), 29-49. MR2097127(2005g:55004)

[11] E. C. Keppelmann and C. K. McCord, The Anosov theorem for exponential solvmanifolds, Pacific J. Math., 170 (1995), 143-159. MR1359975 (96j:55005)

[12] S. W. Kim and J. B. Lee, Anosov theorem for coincidences on nilmanifolds, Fund. Math., 185 (2005), 247-259. MR2161405 (2006e:55005)

[13] J. B. Lee and X. Zhao, Nielsen type numbers and homotopy minimal periods for maps on the 3-solvmanifolds, Algebr. Geom. Topol., 8 (2008), 563-580. MR2443238 (2009g:55002)

[14] C. K. McCord, Lefschetz and Nielsen coincidence numbers on nilmanifolds and solvmanifolds, II, Topology Appl., 75 (1997), 81-92. MR.1425386 (98a:55004)

[15] J. Vick, Homology Theory: An introduction to algebraic topology, second edition, Graduate Texts in Mathematics, 145, Springer-Verlag, New York, 1994. MR1254439 (94i:55002)

[16] P. Wong, Reidemeister number, Hirsch rank, coincidences on polycyclic groups and solvmanifolds, J. reine angew. Math., 524 (2000), 185-204. MR1770607(2002b:57044)

Department of Mathematics, Sogang University, Seoul 121-742, Republic of Korea

E-mail address: kyha@sogang.ac.kr

Department of Mathematics, Sogang University, Seoul 121-742, Republic of Korea

E-mail address: jlee@sogang.ac.kr

Department of Mathematics, Katholieke Universiteit Leuven, Campus Kortrijk, B-8500 KortriJK, Belgium

E-mail address: pieter.penninckx@kuleuven-kortrijk.be 